\title{
NUMERICAL ANALYSIS OF COUPLING FOR A KINETIC EQUATION
}

\author{
MOUlay TIDRIRI ${ }^{1}$
}

\begin{abstract}
In this paper we introduce a coupled systems of kinetic equations for the linearized Carleman model. We then study the existence theory and the asymptotic behaviour of the resulting coupled problem. In order to solve the coupled problem we propose to use the time marching algorithm. We then develop a convergence theory for the resulting algorithm. Numerical results confirming the theory are then presented.
\end{abstract}

AMS Subject Classification. 35Q35, 35L50, 65M12, 82B40, 76P05.

Received: January 26, 1999. Revised: March 4, 1999.

\section{INTRODUCTION}

The coupling of kinetic equations and their hydrodynamic limits was introduced and studied in $[3,12]$ see also $[4,5,8-11,13]$. This approach was introduced in order to solve several difficulties that occur at the interface between fluid mechanics and kinetic theory. Because of the practical importance of these methodologies, the establishment of their mathematical foundations is of crucial importance. The mathematical theory of such coupling started in $[6,7,12]$, where the coupling of two models of hydrodynamical type is considered. In [15], the author provided an analysis of the coupling of two models of kinetic type. In this paper we shall further study the coupling of kinetic equations for the linearized Carleman model. In particular, we shall study the existence theory and the asymptotic behaviour of the resulting coupled systems. To solve the coupled problem, we propose to use the time marching algorithm also introduced in $[6,7,12-14]$. We shall then establish the convergence theory for the resulting algorithm. Finally, we provide numerical results confirming the above mentioned mathematical results.

We consider in this paper the following linearized Carleman system [1].

$$
\begin{aligned}
& \left.\frac{\partial u}{\partial t}+\frac{\partial u}{\partial x}=a(v-u) \quad \text { on }\right] 0,1[, \\
& \left.\frac{\partial v}{\partial t}-\frac{\partial v}{\partial x}=a(u-v) \quad \text { on }\right] 0,1[, \\
& u(0, \cdot)=u_{0}, \quad v(0, \cdot)=v_{0}, \\
& u(t, 0)=g(t), \quad v(t, 1)=h(t),
\end{aligned}
$$

where $t \in \mathbb{R}^{+}, x \in[0,1]$ and $u(t, x), v(t, x)$ are functions of $x$ which represent probability densities for particles moving in the positive and negative $x$ - direction, respectively. $a$ is a positive constant and $g(t)$ and $h(t)$ are

Keywords and phrases. Asymptotic behaviour, Carleman equations, coupling of kinetic equations, time marching algorithm.

1 Department of Mathematics, Iowa State University, Ames, IA 50011-2064, USA. e-mail: tidriri@iastate.edu 
two nonnegative functions. This model describes a random walk in one dimension. System (1-4) has a unique strong solution.

The steady state problem corresponding to equations $(1-4)$ is

$$
\begin{array}{cc}
u^{\prime}=a(v-u) & \text { on }] 0,1[ \\
-v^{\prime}=a(u-v) & \text { on }] 0,1[ \\
u(0)=g, & v(1)=h .
\end{array}
$$

The coupled strategy of $[6,7,12-14]$ applied to system $(1-4)$ leads to the following coupled systems

$$
\begin{gathered}
\left.\frac{\partial u_{1}}{\partial t}+\frac{\partial u_{1}}{\partial x}=a\left(v_{1}-u_{1}\right) \quad \text { on }\right] 0, h_{1}[, \\
\left.\frac{\partial v_{1}}{\partial t}-\frac{\partial v_{1}}{\partial x}=a\left(u_{1}-v_{1}\right) \quad \text { on }\right] 0, h_{1}[, \\
u_{1}(0, \cdot)=u_{10}, \quad v_{1}(0, \cdot)=v_{10}, \\
u_{1}(t, 0)=g(t), \quad v_{1}\left(t, h_{1}\right)=v_{2}\left(t, h_{1}\right),
\end{gathered}
$$

and

$$
\begin{array}{cc}
\frac{\partial u_{2}}{\partial t}+\frac{\partial u_{2}}{\partial x}=a\left(v_{2}-u_{2}\right) & \text { on }] h_{1}, 1[, \\
\frac{\partial v_{2}}{\partial t}-\frac{\partial v_{2}}{\partial x}=a\left(u_{2}-v_{2}\right) & \text { on }] h_{1}, 1[, \\
u_{2}(0, \cdot)=u_{20}, & v_{2}(0, \cdot)=v_{20}, \\
u_{2}\left(t, h_{1}\right)=u_{1}\left(t, h_{1}\right), & v_{2}(t, 1)=h(t),
\end{array}
$$

where $0<h_{1}<1$. Notice that problems $(8-11,12-15)$ are only coupled by their boundary conditions. They can be solved by two independent solution techniques.

In Section 2 and 3, we shall state and prove results about the existence theory and asymptotic behaviour of the coupled systems. In Section 4, we shall study the convergence properties of the time marching algorithm applied to the coupled problem. Finally, in Section 5, we present a numerical study of the resulting algorithm.

\section{EXISTENCE THEORY}

In this section, we shall study the existence of a solution for the coupled problem introduced in the previous section. We shall work in the Hilbert space

$$
X=\left(L^{2}\left[0, h_{1}\right]\right)^{2} \times\left(L^{2}\left[h_{1}, 1\right]\right)^{2},
$$

with the following norm

$$
\left\|\left(w_{1}, w_{2}, w_{3}, w_{4}\right)\right\|=\left(\left\|w_{1}\right\|_{L^{2}\left[0, h_{1}\right]}^{2}+\left\|w_{2}\right\|_{L^{2}\left[0, h_{1}\right]}^{2}+\left\|w_{3}\right\|_{L^{2}\left[h_{1}, 1\right]}^{2}+\left\|w_{4}\right\|_{L^{2}\left[h_{1}, 1\right]}^{2}\right)^{\frac{1}{2}}
$$

The main result of this section is the following

Theorem 2.1. Assume that $\left(u_{10}, v_{10}, u_{20}, v_{20}\right) \in X$, then the coupled problem (8-15) has a unique strong solution $\left(u_{1}, v_{1}, u_{2}, v_{2}\right)$. 
We shall give the proof of this theorem for the homogeneous boundary conditions: $g(t)=0$ and $h(t)=0$. By a standard argument the proof in the nonhomogeneous case can be reduced to the homogeneous case.

We introduce an operator $A$ on $X$ as follows

$$
\begin{aligned}
A\left(w_{1}, w_{2}, w_{3}, w_{4}\right) & =\left(\begin{array}{c}
w_{1}^{\prime}+a\left(w_{1}-w_{2}\right) \\
-w_{2}^{\prime}+a\left(w_{2}-w_{1}\right) \\
w_{3}^{\prime}+a\left(w_{3}-w_{4}\right) \\
-w_{4}^{\prime}+a\left(w_{4}-w_{3}\right)
\end{array}\right), \\
D(A) & =\left\{\begin{array}{c}
\left(w_{1}, w_{2}, w_{3}, w_{4}\right) \in X \mid w_{1}^{\prime}, w_{2}^{\prime} \in L^{2}\left[0, h_{1}\right], w_{3}^{\prime}, \text { and } w_{4}{ }^{\prime} \in L^{2}\left[h_{1}, 1\right] \\
w_{1}(0)=0, w_{4}(1)=0,\left(w_{1}-w_{3}\right)\left(h_{1}\right)=0 \text { and }\left(w_{2}-w_{4}\right)\left(h_{1}\right)=0
\end{array}\right\} .
\end{aligned}
$$

It is clear that $D(A)$ is dense in $X$. We shall first, prove that the coupled problem has a unique solution in $D(A)$. This will be a consequence of the following theorem which establishes that the operator $-A$ is the infinitesimal generator of a contraction semigroup of class $\mathcal{C}^{0}$.

Theorem 2.2. The operator $-A$ is the infinitesimal generator of a contraction semigroup of class $\mathcal{C}^{0}$ : $\{G(t) ; t \geq 0\}$.

Remark 1. As a consequence of Theorem (2.2) the existence theory is established.

Proof. To prove Theorem (2.2), we shall use the following result of Hille-Phillips [2].

Theorem 2.3. Let $T$ be an unbounded operator with domain $D(T)$ dense in the Hilbert space $X$. Then $T$ is the infinitesimal generator of a contraction semigroup of class $\mathcal{C}^{0}$ if and only if

(i) $T$ is dissipative;

(ii) the range of $D(T)$ by $I-T$ is equal to $X$.

Let $T=-A$, where $A$ is the unbounded operator defined in (16). Let $w$ be an element of $D(A)$. $T$ satisfies

$$
\begin{aligned}
(T w, w)= & -(A w, w) \\
= & \int_{0}^{h_{1}}\left(-w_{1}^{\prime}+a\left(w_{2}-w_{1}\right)\right) w_{1}+\int_{0}^{h_{1}}\left(w_{2}^{\prime}+a\left(w_{1}-w_{2}\right)\right) w_{2}+\int_{h_{1}}^{1}\left(-w_{3}^{\prime}+a\left(w_{4}-w_{3}\right)\right) w_{3} \\
& +\int_{h_{1}}^{1}\left(w_{4}^{\prime}+a\left(w_{3}-w_{4}\right)\right) w_{4} \\
= & -\frac{1}{2}\left(w_{2}^{2}(0)+w_{3}^{2}(1)\right)-a \int_{0}^{h_{1}}\left(w_{1}-w_{2}\right)^{2}-a \int_{h_{1}}^{1}\left(w_{3}-w_{4}\right)^{2} .
\end{aligned}
$$

To obtain the last equality, we have used the boundary conditions. Since $a>0$, we have $(T u, u) \leq 0$ and $-A$ is dissipative.

Next, we show that the range of $D(T)$ by $I-T$ is equal to $X$. Let $f \in X$ and consider the problem of finding $u \in D(T)$ such that

$$
(I-T) w=f,
$$

which corresponds to finding $w \in D(T)$ such that

$$
\begin{aligned}
w_{1}^{\prime}+(a+1) w_{1}-a w_{2} & =f_{1}, \\
-w_{2}^{\prime}+(a+1) w_{2}-a w_{1} & =f_{2}, \\
w_{3}^{\prime}+(a+1) w_{3}-a w_{4} & =f_{3}, \\
-w_{4}^{\prime}+(a+1) w_{4}-a w_{3} & =f_{4} .
\end{aligned}
$$


By a density argument we may assume that $f_{1}, f_{2}, f_{3}$, and $f_{4}$ are continuous. By elementary methods we obtain the general solution of system (19-20). Similarly, we obtain the general solution of system (21-22). By using the boundary conditions we conclude that problem (18) has a unique solution. The use of Theorem 2.3 concludes the proof of the theorem.

\section{Asymptotic ANALYSis OF THE COUPLED SySTEMS}

The main result of this section is

Theorem 3.1. Assume that $u_{10}, v_{10} \in L^{2}\left[0, h_{1}\right]$ and $u_{20}, v_{20} \in L^{2}\left[h_{1}, 1\right]$, then the solution of the coupled problem (8-15) converges as $t$ tends to $+\infty$ to the solution of the steady problem (5-7).

Proof. As in the previous section, without loss of generality, we may assume that $g=g(t)=0$ and $h=h(t)=0$. Let $\left(u_{s}, v_{s}\right)$ denote the solution of the steady problem (5-7). Let $\bar{u}$ and $\bar{v}$ be defined as follows

$$
\begin{aligned}
& \left.\bar{u}=u_{1}-u_{s} \text { and } \bar{v}=v_{1}-v_{s} \quad \text { on }\right] 0, h_{1}[\text {, } \\
& \left.\bar{u}=u_{2}-u_{s} \text { and } \bar{v}=v_{2}-v_{s} \text { on }\right] h_{1}, 1[\text {, }
\end{aligned}
$$

where $\left(u_{1}, v_{1}, u_{2}, v_{2}\right)$ is the solution of the coupled problem (8-15). Then we have

$$
\begin{array}{ll}
\frac{\partial \bar{u}_{1}}{\partial t}+\frac{\partial \bar{u}_{1}}{\partial x}=a\left(\bar{v}_{1}-\bar{u}_{1}\right) & \text { on }] 0, h_{1}[, \\
\frac{\partial \bar{v}_{1}}{\partial t}-\frac{\partial \bar{v}_{1}}{\partial x}=a\left(\bar{u}_{1}-\bar{v}_{1}\right) & \text { on }] 0, h_{1}[, \\
\bar{u}_{1}(0, \cdot)=u_{10}, & \bar{v}_{1}(0, \cdot)=v_{10}, \\
\bar{u}_{1}(t, 0)=0, & \bar{v}_{1}\left(t, h_{1}\right)=\bar{v}_{2}\left(t, h_{1}\right),
\end{array}
$$

and

$$
\begin{array}{cc}
\frac{\partial \bar{u}_{2}}{\partial t}+\frac{\partial \bar{u}_{2}}{\partial x}=a\left(\bar{v}_{2}-\bar{u}_{2}\right) & \text { on }] h_{1}, 1[ \\
\frac{\partial \bar{v}_{2}}{\partial t}-\frac{\partial \bar{v}_{2}}{\partial x}=a\left(\bar{u}_{2}-\bar{v}_{2}\right) & \text { on }] h_{1}, 1[ \\
\bar{u}_{2}(0, \cdot)=u_{20}, & \bar{v}_{2}(0, \cdot)=v_{20} \\
\bar{u}_{2}\left(t, h_{1}\right)=\bar{u}_{1}\left(t, h_{1}\right), & \bar{v}_{2}(t, 1)=0 .
\end{array}
$$

Let $w=\left(w_{1}, w_{2}, w_{3}, w_{4}\right)=\left(\bar{u}_{1}, \bar{v}_{1}, \bar{u}_{2}, \bar{v}_{2}\right)$. Let $\varphi_{1}$ and $\varphi_{2}$ be two positive functions independent of $t$ to be precised later. Multiplying equations $(23,24)$ and $(27,28)$ respectively by $\varphi_{1} w_{1}, \varphi_{2} w_{2}$ and $\varphi_{1} w_{3}, \varphi_{2} w_{4}$, integrating over $\left[0, h_{1}\right]$ respectively $\left[h_{1}, 1\right]$, and using Cauchy-Schwarz inequality, we obtain

$$
\begin{aligned}
\frac{\mathrm{d}}{\mathrm{d} t} \int_{0}^{h_{1}}\left(\varphi_{1} w_{1}^{2}+\varphi_{2} w_{2}^{2}\right)+\int_{0}^{h_{1}}\left(-\varphi_{1 x}+\right. & \left.a\left(\varphi_{1}-\varphi_{2}\right)\right) w_{1}^{2}+\int_{0}^{h_{1}}\left(\varphi_{2 x}+a\left(\varphi_{2}-\varphi_{1}\right)\right) w_{2}^{2} \\
& +\varphi_{1}\left(h_{1}\right) w_{1}^{2}\left(h_{1}\right)-\varphi_{1}(0) w_{1}^{2}(0)-\varphi_{2}\left(h_{1}\right) w_{2}^{2}\left(h_{1}\right)+\varphi_{2}(0) w_{2}^{2}(0) \leq 0
\end{aligned}
$$

and

$$
\begin{aligned}
\frac{\mathrm{d}}{\mathrm{d} t} \int_{h_{1}}^{1}\left(\varphi_{1} w_{3}^{2}+\varphi_{2} w_{4}^{2}\right)+\int_{h_{1}}^{1}\left(-\varphi_{1 x}+a\left(\varphi_{1}-\varphi_{2}\right)\right) w_{3}^{2}+\int_{h_{1}}^{1}\left(\varphi_{2 x}+a\left(\varphi_{2}-\varphi_{1}\right)\right) w_{4}^{2} \\
\\
\quad+\varphi_{1}(1) w_{3}^{2}(1)-\varphi_{1}\left(h_{1}\right) w_{3}^{2}\left(h_{1}\right)-\varphi_{2}(1) w_{4}^{2}(1)+\varphi_{2}\left(h_{1}\right) w_{4}^{2}\left(h_{1}\right) \leq 0 .
\end{aligned}
$$


Assuming that $\varphi_{1}\left(h_{1}\right)=\varphi_{2}\left(h_{1}\right)$, and using the boundary conditions, we obtain

$$
\begin{aligned}
\frac{\mathrm{d}}{\mathrm{d} t} \int_{0}^{h_{1}}\left(\varphi_{1} w_{1}^{2}+\varphi_{2} w_{2}^{2}\right) & +\int_{0}^{h_{1}}\left(-\varphi_{1 x}+a\left(\varphi_{1}-\varphi_{2}\right)\right) w_{1}^{2}+\int_{0}^{h_{1}}\left(\varphi_{2 x}+a\left(\varphi_{2}-\varphi_{1}\right)\right) w_{2}^{2} \\
& +\frac{\mathrm{d}}{\mathrm{d} t} \int_{h_{1}}^{1}\left(\varphi_{1} w_{3}^{2}+\varphi_{2} w_{4}^{2}\right)+\int_{h_{1}}^{1}\left(-\varphi_{1 x}+a\left(\varphi_{1}-\varphi_{2}\right)\right) w_{3}^{2}+\int_{h_{1}}^{1}\left(\varphi_{2 x}+a\left(\varphi_{2}-\varphi_{1}\right)\right) w_{4}^{2} \leq 0
\end{aligned}
$$

Now consider the following system of equations

$$
\begin{aligned}
-\varphi_{1 x}+a \varphi_{1}-a \varphi_{2} & =k \text { on }] 0, h_{1}\left[(\text { resp. on }] h_{1}, 1[)\right. \\
\varphi_{2 x}+a \varphi_{2}-a \varphi_{1} & =k \text { on }] 0, h_{1}\left[(\text { resp. on }] h_{1}, 1[)\right. \\
\varphi_{1}\left(h_{1}\right) & =\varphi_{2}\left(h_{1}\right)
\end{aligned}
$$

where $k$ is a positive constant. The solution of this system is given by

$$
\begin{aligned}
& \varphi_{1}(x)=\varphi_{1}\left(h_{1}\right)+\left(h_{1}-x\right) k-a k\left(h_{1}-x\right)^{2}, \\
& \varphi_{2}(x)=\varphi_{1}\left(h_{1}\right)+\left(x-h_{1}\right) k-a k\left(h_{1}-x\right)^{2} .
\end{aligned}
$$

Choosing for example $\varphi_{1}\left(h_{1}\right)=2 a h_{1}^{2} k+2 h_{1} k+(3+a) k$, we obtain

$$
\begin{array}{ll}
k<\varphi_{1}(x) \leq K & \left.\forall x \in\left[0, h_{1}\right] \quad \text { (resp. on }\left[h_{1}, 1\right]\right) \\
k<\varphi_{2}(x) \leq K & \left.\forall x \in\left[0, h_{1}\right] \quad \text { (resp. on }\left[h_{1}, 1\right]\right)
\end{array}
$$

where $K$ is a positive constant independent of $h_{1}$.

Combining $(33,34,35)$, we then obtain

$$
\frac{\mathrm{d}}{\mathrm{d} t}\left[\int_{0}^{h_{1}}\left(\varphi_{1} w_{1}^{2}+\varphi_{2} w_{2}^{2}\right)+\int_{h_{1}}^{1}\left(\varphi_{1} w_{3}^{2}+\varphi_{2} w_{4}^{2}\right)\right]+\int_{0}^{h_{1}}\left(f_{1} w_{1}^{2}+f_{2} w_{2}^{2}\right)+\int_{h_{1}}^{1}\left(f_{1} w_{3}^{2}+f_{2} w_{4}^{2}\right) \leq 0
$$

Using the properties of $\varphi_{1}$ and $\varphi_{2}$ we finally obtain

$$
\int_{0}^{h_{1}}\left(w_{1}^{2}+w_{2}^{2}\right)+\int_{h_{1}}^{1}\left(w_{3}^{2}+w_{4}^{2}\right) \leq \mathrm{e}^{-c_{0} t}\left[\int_{0}^{h_{1}}\left(w_{10}^{2}+w_{20}^{2}\right)+\int_{h_{1}}^{1}\left(w_{30}^{2}+w_{40}^{2}\right)\right]
$$

And this concludes the proof of Theorem 3.1. 


\section{Numerical analysis of the COUPling}

To solve the coupled problem introduced in previous sections, we propose to use the time marching algorithm of $[6,7,12-14]$. In this section we shall study the convergence properties of the algorithm.

$$
\begin{aligned}
& \left.\frac{u_{1}^{n+1}-u_{1}^{n}}{\Delta t}+\frac{\mathrm{d} u_{1}^{n+1}}{\mathrm{~d} x}=a\left(v_{1}^{n+1}-u_{1}^{n+1}\right) \quad \text { on }\right] 0, h_{1}[, \\
& \left.\frac{v_{1}^{n+1}-v_{1}^{n}}{\Delta t}-\frac{\mathrm{d} v_{1}^{n+1}}{\mathrm{~d} x}=a\left(u_{1}^{n+1}-v_{1}^{n+1}\right) \quad \text { on }\right] 0, h_{1}[, \\
& u_{1}^{n+1}(0)=0, \quad v_{1}^{n+1}\left(h_{1}\right)=v_{2}^{n+1}\left(h_{1}\right), \\
& \left.\frac{u_{2}^{n+1}-u_{2}^{n}}{\Delta t}+\frac{\mathrm{d} u_{2}^{n+1}}{\mathrm{~d} x}=a\left(v_{2}^{n+1}-u_{2}^{n+1}\right) \quad \text { on }\right] h_{1}, 1[, \\
& \left.\frac{v_{2}^{n+1}-v_{2}^{n}}{\Delta t}-\frac{\mathrm{d} v_{2}^{n+1}}{\mathrm{~d} x}=a\left(u_{2}^{n+1}-v_{2}^{n+1}\right) \quad \text { on }\right] h_{1}, 1[, \\
& u_{2}^{n+1}\left(h_{1}\right)=u_{1}^{n}\left(h_{1}\right), \quad v_{2}^{n+1}(1)=0,
\end{aligned}
$$

and the initial conditions

$$
u_{1}^{0}=u_{10}, \quad v_{1}^{0}=v_{10}, \quad u_{2}^{0}=u_{20}, \quad v_{2}^{0}=v_{20} .
$$

Here, without loss of generality, we have assumed that $g=g(t)=0$ and $h=h(t)=0$.

The convergence of the algorithm (41-47) is stated in the following theorem.

Theorem 4.1. The algorithm (41-47) converges as $n$ tends to $\infty$.

The proof of this theorem will be given in Section 4.2 .

\subsection{The time independent case}

If we introduce the coupled systems directly for the steady problem and we apply the time marching algorithm, we obtain

$$
\begin{gathered}
\left.\frac{\mathrm{d} u_{1}^{n+1}}{\mathrm{~d} x}=a\left(v_{1}^{n+1}-u_{1}^{n+1}\right) \quad \text { on }\right] 0, h_{1}[, \\
\left.-\frac{\mathrm{d} v_{1}^{n+1}}{\mathrm{~d} x}=a\left(u_{1}^{n+1}-v_{1}^{n+1}\right) \quad \text { on }\right] 0, h_{1}[, \\
u_{1}^{n+1}(0)=0, \quad v_{1}^{n+1}\left(h_{1}\right)=v_{2}^{n+1}\left(h_{1}\right), \\
\left.\frac{\mathrm{d} u_{2}^{n+1}}{\mathrm{~d} x}=a\left(v_{2}^{n+1}-u_{2}^{n+1}\right) \quad \text { on }\right] h_{1}, 1[, \\
\left.-\frac{\mathrm{d} v_{2}^{n+1}}{\mathrm{~d} x}=a\left(u_{2}^{n+1}-v_{2}^{n+1}\right) \quad \text { on }\right] h_{1}, 1[, \\
u_{2}^{n+1}\left(h_{1}\right)=u_{1}^{n}\left(h_{1}\right), \quad v_{2}^{n+1}(1)=0, \\
u_{1}^{0}=u_{10}, \quad v_{1}^{0}=v_{10}, \quad u_{2}^{0}=u_{20}, \quad v_{2}^{0}=v_{20} .
\end{gathered}
$$

The following theorem states the convergence of the algorithm (48-54). 
Theorem 4.2. The algorithm (48-54) converges for any $0<h_{1}<1$.

Proof. We first set

$$
u_{2}^{(n)}\left(h_{1}\right)=\gamma^{(n)}, \quad v_{1}^{(n)}\left(h_{1}\right)=\delta^{(n)}
$$

The algorithm (48-54) converges if $\gamma^{(n)} \rightarrow 0$ and $\delta^{(n)} \rightarrow 0$ as $n \rightarrow \infty$. The solution of problem (48-50) is given by

$$
u_{1}^{(n+1)}(x)=\frac{v_{2}^{(n+1)}\left(h_{1}\right)}{1+a h_{1}} a x, \quad v_{1}^{(n+1)}(x)=\frac{v_{2}^{(n+1)}\left(h_{1}\right)}{1+a h_{1}}(1+a x) .
$$

The solution of problem (51-53) is given by

$$
u_{2}^{(n+1)}(x)=\frac{u_{1}^{(n)}\left(h_{1}\right)}{1+a\left(1-h_{1}\right)}(1+a(1-x)), \quad v_{2}^{(n+1)}(x)=\frac{u_{1}^{(n)}\left(h_{1}\right)}{1+a\left(1-h_{1}\right)} a(1-x) .
$$

The solution of the steady problem (5-6) with boundary conditions $u(0)=0$ and $v(1)=0$ is $u=0$ and $v=0$.

Using the coupling boundary conditions, we obtain

$$
\begin{aligned}
\gamma^{(1)} & =u_{2}^{(1)}\left(h_{1}\right)=u_{1}^{(0)}\left(h_{1}\right) \\
\delta^{(1)} & =v_{1}^{(1)}\left(h_{1}\right)=v_{2}^{(1)}\left(h_{1}\right) \\
& =\frac{a\left(1-h_{1}\right)}{1+a\left(1-h_{1}\right)} \gamma^{(1)} .
\end{aligned}
$$

On the other hand using the coupling boundary conditions, we obtain

$$
\begin{aligned}
\gamma^{(2)} & =u_{2}^{(2)}\left(h_{1}\right)=u_{1}^{(1)}\left(h_{1}\right) \\
& =\frac{a h_{1}}{1+a h_{1}} \frac{a\left(1-h_{1}\right)}{1+a\left(1-h_{1}\right)} \gamma^{(1)}, \\
\delta^{(2)} & =v_{1}^{(2)}\left(h_{1}\right)=v_{2}^{(2)}\left(h_{1}\right) \\
& =\frac{a\left(1-h_{1}\right)}{1+a\left(1-h_{1}\right)} \frac{a h_{1}}{1+a h_{1}} \delta^{(1)} .
\end{aligned}
$$

Hence, the iterative process is completely determined

$$
\begin{aligned}
\gamma^{(n+1)} & =\frac{a h_{1}}{1+a h_{1}} \frac{a\left(1-h_{1}\right)}{1+a\left(1-h_{1}\right)} \gamma^{(n)}, \\
\delta^{(n+1)} & =\frac{a\left(1-h_{1}\right)}{1+a\left(1-h_{1}\right)} \frac{a h_{1}}{1+a h_{1}} \delta^{(n)} .
\end{aligned}
$$

From the last iterative process, we clearly deduce the convergence of the algorithm. And this concludes the proof of Theorem 4.2.

\subsection{Analysis of the general algorithm}

We shall now establish the convergence properties of the algorithm (41-47). More precisely, we shall give the proof of Theorem 4.1 . 
Proof of Theorem 4.1. Using the notation $u_{1}^{n+1}=u_{1}, \quad v_{1}^{n+1}=v_{1}, \quad u_{1}^{n}=f_{1}, \quad v_{1}^{n}=g_{1}, u_{2}^{n+1}=u_{2}, \quad v_{2}^{n+1}=$ $v_{2}, \quad u_{2}^{n}=f_{2}, \quad v_{2}^{n}=g_{2}$, the algorithm $(41-47)$ becomes

$$
\begin{gathered}
\left\{\begin{array}{l}
\left.b u_{1}+u_{1}^{\prime}=a v_{1}+c f_{1} \quad \text { on }\right] 0, h_{1}[, \\
\left.b v_{1}-v_{1}^{\prime}=a u_{1}+c g_{1} \quad \text { on }\right] 0, h_{1}[, \\
u_{1}(0)=0, \quad v_{1}\left(h_{1}\right)=v_{2}\left(h_{1}\right),
\end{array}\right. \\
\left\{\begin{array}{l}
\left.b u_{2}+u_{2}^{\prime}=a v_{2}+c f_{2} \quad \text { on }\right] h_{1}, 1[, \\
\left.b v_{2}-v_{2}^{\prime}=a u_{2}+c g_{2} \quad \text { on }\right] h_{1}, 1[, \\
u_{2}\left(h_{1}\right)=f_{1}\left(h_{1}\right), \quad v_{2}(1)=0,
\end{array}\right.
\end{gathered}
$$

with the initial conditions (47). Here, we have used the notation $b=a+1 / \Delta t$ and $c=1 / \Delta t$.

Let $\varphi_{1}, \varphi_{2}, \varphi_{3}$ and $\varphi_{4}$ be four positive functions bounded below and above to be precised later. Multiplying equations $(58,59)$ respectively by $\varphi_{1}, \varphi_{2}, \varphi_{3}$ and $\varphi_{4}$, integrating respectively over $\left[0, h_{1}\right]$ and $\left[h_{1}, 1\right]$ and using Cauchy-Schwarz inequality we obtain

$$
\int_{0}^{h_{1}}\left[\frac{a+c}{2} \varphi_{1}-\frac{1}{2} \varphi_{1}^{\prime}-\frac{a}{2} \varphi_{2}\right] u_{1}^{2}+\int_{0}^{h_{1}}\left[\frac{a+c}{2} \varphi_{2}+\frac{1}{2} \varphi_{2}^{\prime}-\frac{a}{2} \varphi_{1}\right] v_{1}^{2}+\frac{1}{2}\left(\varphi_{1} u_{1}^{2}-\varphi_{2} v_{1}^{2}\right)_{0}^{h_{1}} \leq \frac{c}{2} \int_{0}^{h_{1}}\left(\varphi_{1} f_{1}^{2}+\varphi_{2} g_{1}^{2}\right)
$$

and

$$
\int_{h_{1}}^{1}\left[\frac{a+c}{2} \varphi_{3}-\frac{1}{2} \varphi_{3}^{\prime}-\frac{a}{2} \varphi_{4}\right] u_{2}^{2}+\int_{h_{1}}^{1}\left[\frac{a+c}{2} \varphi_{4}+\frac{1}{2} \varphi_{4}^{\prime}-\frac{a}{2} \varphi_{3}\right] v_{2}^{2}+\frac{1}{2}\left(\varphi_{3} u_{2}^{2}-\varphi_{4} v_{2}^{2}\right)_{h_{1}}^{1} \leq \frac{c}{2} \int_{h_{1}}^{1}\left(\varphi_{3} f_{2}^{2}+\varphi_{4} g_{2}^{2}\right) .
$$

Using the boundary conditions and combining $(60,61)$, we obtain

$$
\begin{array}{r}
\int_{0}^{h_{1}}\left[\frac{a+c}{2} \varphi_{1}-\frac{1}{2} \varphi_{1}^{\prime}-\frac{a}{2} \varphi_{2}\right] u_{1}^{2}+\int_{0}^{h_{1}}\left[\frac{a+c}{2} \varphi_{2}+\frac{1}{2} \varphi_{2}^{\prime}-\frac{a}{2} \varphi_{1}\right] v_{1}^{2}+\int_{h_{1}}^{1}\left[\frac{a+c}{2} \varphi_{3}-\frac{1}{2} \varphi_{3}^{\prime}-\frac{a}{2} \varphi_{4}\right] u_{2}^{2}+\int_{h_{1}}^{1}\left[\frac{a+c}{2} \varphi_{4}\right. \\
\left.+\frac{1}{2} \varphi_{4}^{\prime}-\frac{a}{2} \varphi_{3}\right] v_{2}^{2}+\frac{1}{2} \varphi_{1}\left(h_{1}\right) u_{1}^{2}\left(h_{1}\right)+\frac{1}{2}\left[\varphi_{4}\left(h_{1}\right) v_{2}^{2}\left(h_{1}\right)-\varphi_{2}\left(h_{1}\right) v_{1}^{2}\left(h_{1}\right)\right]+\frac{1}{2} \varphi_{2}(0) v_{1}^{2}(0)+\frac{1}{2} \varphi_{3}(1) u_{2}^{2}(1) \\
\leq \frac{c}{2} \int_{0}^{h_{1}}\left(\varphi_{1} f_{1}^{2}+\varphi_{2} g_{1}^{2}\right)+\frac{c}{2} \int_{h_{1}}^{1}\left(\varphi_{3} f_{2}^{2}+\varphi_{4} g_{2}^{2}\right)+\frac{1}{2} \varphi_{3}\left(h_{1}\right) f_{1}^{2}\left(h_{1}\right) .
\end{array}
$$

Now our objective is to find $\gamma_{1}$ and $\gamma_{2}$ both larger than $c / 2$ such that the solutions $\varphi_{1}$ and $\varphi_{2}$ of the following system

$$
\begin{aligned}
& \left.\frac{a+c}{2} \varphi_{1}-\frac{1}{2} \varphi_{1}^{\prime}-\frac{a}{2} \varphi_{2}=\gamma_{1} \varphi_{1} \text { on }\right] 0, h_{1}[ \\
& \left.\frac{a+c}{2} \varphi_{2}+\frac{1}{2} \varphi_{2}^{\prime}-\frac{a}{2} \varphi_{1}=\gamma_{2} \varphi_{2} \text { on }\right] 0, h_{1}[
\end{aligned}
$$

with the boundary conditions $\varphi_{1}\left(h_{1}\right)$ and $\varphi_{2}\left(h_{1}\right)$ fixed and positive to be precised later, are both positive bounded below and above by positive constants which are independent of both $c$ and $h_{1}$. By choosing $\gamma_{1}=$ $\gamma_{2}=(c+\epsilon) / 2$ and setting $\lambda=a-\epsilon$ where $\epsilon$ is a small positive parameter, we find

$$
\begin{aligned}
\varphi_{1} & =\frac{1}{a} \mathrm{e}^{-\lambda x} v^{\prime}, \quad \varphi_{2}=\mathrm{e}^{-\lambda x} v \\
v & =\mathrm{e}^{\lambda x}\left(c_{1} \cos \sqrt{\epsilon(\lambda+a)} x+c_{2} \sin \sqrt{\epsilon(\lambda+a)} x\right),
\end{aligned}
$$


with $c_{1}$ and $c_{2}$ given by

$$
\begin{aligned}
c_{1} & =\frac{a \mathrm{e}^{\alpha_{2} h_{1}} \varphi_{1}\left(h_{1}\right)-\frac{b_{2}}{a_{2}} \mathrm{e}^{\alpha_{2} h_{1}} \varphi_{2}\left(h_{1}\right)}{b_{1}-\frac{a_{1}}{a_{2}} b_{2}}, \\
c_{2} & =\frac{1}{a_{2}} \mathrm{e}^{\alpha_{2} h_{1}} \varphi_{2}\left(h_{1}\right)-\frac{a_{1}}{a_{2}} \frac{a \mathrm{e}^{\alpha_{2} h_{1}} \varphi_{1}\left(h_{1}\right)-\frac{b_{2}}{a_{2}} \mathrm{e}^{\alpha_{2} h_{1}} \varphi_{2}\left(h_{1}\right)}{b_{1}-\frac{a_{1}}{a_{2}} b_{2}} .
\end{aligned}
$$

Here, $a_{1}, a_{2}, b_{1}$ and $b_{2}$ are given by

$$
\begin{aligned}
a_{1} & =\mathrm{e}^{\lambda h_{1}} \cos \sqrt{\epsilon(\lambda+a)} h_{1}, \quad a_{2}=\mathrm{e}^{\lambda h_{1}} \sin \sqrt{\epsilon(\lambda+a)} h_{1} \\
b_{1} & =\lambda a_{1}-\sqrt{\epsilon(\lambda+a)} a_{2} \\
b_{2} & =\lambda a_{2}+\sqrt{\epsilon(\lambda+a)} a_{1} .
\end{aligned}
$$

Assuming that the boundary conditions satisfy

$$
\varphi_{1}\left(h_{1}\right)=\left(1+\frac{\delta}{a}\right) \varphi_{2}\left(h_{1}\right)
$$

with $\delta$ a small positive parameter, we find that $c_{1}, c_{2}, \varphi_{1}$ and $\varphi_{2}$ are positive. Moreover, they satisfy the following inequalities

$$
\begin{aligned}
& C_{1} \varphi_{2}\left(h_{1}\right) \leq \varphi_{1}(x) \leq C_{2} \varphi_{2}\left(h_{1}\right) \\
& C_{3} \varphi_{2}\left(h_{1}\right) \leq \varphi_{2}(x) \leq C_{4} \varphi_{2}\left(h_{1}\right)
\end{aligned}
$$

where $C_{1}, C_{2}, C_{3}$ and $C_{4}$ are positive constants. Using (67) and with an appropriate choice for $\varphi_{2}\left(h_{1}\right)$, we conclude that for $\epsilon$ and $\delta$ small $\varphi_{1}$ and $\varphi_{2}$ are bounded below and above by positive constants which are independent of $c$ and $h_{1}$.

We shall now find $\gamma_{3}$ and $\gamma_{4}$ both larger than $c / 2$ such that the solutions $\varphi_{3}$ and $\varphi_{4}$ of the following system

$$
\begin{aligned}
\frac{a+c}{2} \varphi_{3}-\frac{1}{2} \varphi_{3}^{\prime}-\frac{a}{2} \varphi_{4} & \left.=\gamma_{3} \varphi_{3} \quad \text { on }\right] h_{1}, 1[ \\
\frac{a+c}{2} \varphi_{4}+\frac{1}{2} \varphi_{4}^{\prime}-\frac{a}{2} \varphi_{3} & \left.=\gamma_{4} \varphi_{4} \quad \text { on }\right] h_{1}, 1[, \\
\varphi_{3}\left(h_{1}\right) & =\left(1-\frac{\delta}{a}\right) \varphi_{1}\left(h_{1}\right), \quad \varphi_{4}\left(h_{1}\right)=\varphi_{2}\left(h_{1}\right),
\end{aligned}
$$

are positive bounded below and above by positive constants which are independent of both $c$ and $h_{1}$. We shall choose $\gamma_{3}=\gamma_{4}=(c+\epsilon) / 2$ where $\epsilon$ is a small positive parameter. Setting $\lambda=a-\epsilon$, we find

$$
\varphi_{3}=\frac{1}{a} e^{-\lambda x} v^{\prime}, \quad \varphi_{4}=e^{-\lambda x} v
$$

where $v$ has similar form as in (66).

Proceeding as for $\varphi_{1}$ and $\varphi_{2}$ and using the boundary conditions (70), we find that for $\epsilon$ and $\delta$ small $\varphi_{3}$ and $\varphi_{4}$ are both positive bounded below and above by positive constants which are independent of both $c$ and $h_{1}$. In fact, we may assume that $\epsilon$ and $\delta$ introduced here are the same as those introduced for the construction of $\varphi_{1}$ and $\varphi_{2}$. We then have $\gamma_{1}=\gamma_{2}=\gamma_{3}=\gamma_{4}$. 


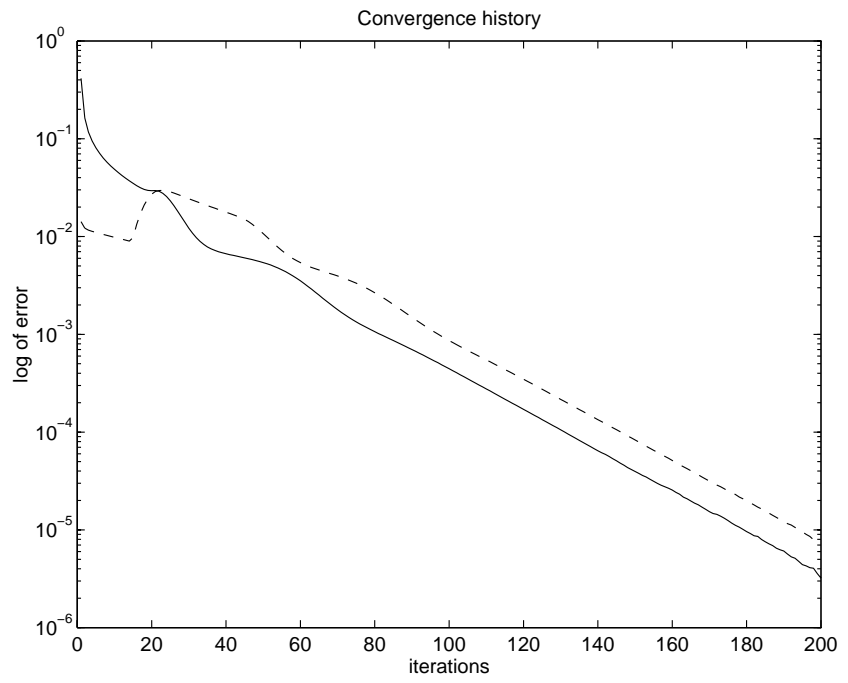

Figure 1. $L_{\infty}$ norm of the error on $\left[0, h_{1}\right](-)$ and $\left[h_{1}, 1\right](--)$.

Using $(63,64,68,69),(62)$ becomes

$$
\begin{gathered}
\gamma_{1} \int_{0}^{h_{1}}\left(\varphi_{1} u_{1}^{2}+\varphi_{2} v_{1}^{2}\right)+\gamma_{1} \int_{h_{1}}^{1}\left(\varphi_{3} u_{2}^{2}+\varphi_{4} v_{2}^{2}\right)+\frac{1}{2} \varphi_{1}\left(h_{1}\right) u_{1}^{2}\left(h_{1}\right)+\frac{1}{2}\left[\varphi_{4}\left(h_{1}\right) v_{2}^{2}\left(h_{1}\right)-\varphi_{2}\left(h_{1}\right) v_{1}^{2}\left(h_{1}\right)\right] \\
+\frac{1}{2} \varphi_{2}(0) v_{1}^{2}(0)+\frac{1}{2} \varphi_{3}(1) u_{2}^{2}(1) \leq \frac{c}{2} \int_{0}^{h_{1}}\left(\varphi_{1} f_{1}^{2}+\varphi_{2} g_{1}^{2}\right)+\frac{c}{2} \int_{h_{1}}^{1}\left(\varphi_{3} f_{2}^{2}+\varphi_{4} g_{2}^{2}\right)+\frac{1}{2} \varphi_{3}\left(h_{1}\right) f_{1}^{2}\left(h_{1}\right) .
\end{gathered}
$$

Using the boundary conditions in $(58,70)$ and the positivity of $\varphi_{2}$ and $\varphi_{3}$, we obtain

$$
\begin{aligned}
\gamma_{1} \int_{0}^{h_{1}}\left(\varphi_{1} u_{1}^{2}+\varphi_{2} v_{1}^{2}\right)+\gamma_{1} \int_{h_{1}}^{1}\left(\varphi_{3}\right. & \left.u_{2}^{2}+\varphi_{4} v_{2}^{2}\right)+\frac{1}{2} \varphi_{1}\left(h_{1}\right) u_{1}^{2}\left(h_{1}\right) \\
& \leq \frac{c}{2} \int_{0}^{h_{1}}\left(\varphi_{1} f_{1}^{2}+\varphi_{2} g_{1}^{2}\right)+\frac{c}{2} \int_{h_{1}}^{1}\left(\varphi_{3} f_{2}^{2}+\varphi_{4} g_{2}^{2}\right)+\frac{1}{2}\left(1-\frac{\delta}{a}\right) \varphi_{1}\left(h_{1}\right) f_{1}^{2}\left(h_{1}\right)
\end{aligned}
$$

Because $\gamma_{1}>c / 2$ and the fact that $\delta$ is small, the operator in (73) is contractant and therefore

$$
\int_{0}^{h_{1}}\left(\varphi_{1} u_{1}^{2}+\varphi_{2} v_{1}^{2}\right)+\int_{h_{1}}^{1}\left(\varphi_{3} u_{2}^{2}+\varphi_{4} v_{2}^{2}\right)+\frac{1}{2 \gamma_{1}} \varphi_{1}\left(h_{1}\right) u_{1}^{2}\left(h_{1}\right),
$$

converges to 0 . Because of our special construction of the functions $\varphi_{1}, \varphi_{2}, \varphi_{3}$ and $\varphi_{4}$, we conclude that

$$
\int_{0}^{h_{1}}\left(u_{1}^{2}+v_{1}^{2}\right)+\int_{h_{1}}^{1}\left(u_{2}^{2}+v_{2}^{2}\right)+\frac{1}{2 \gamma_{1}} u_{1}^{2}\left(h_{1}\right),
$$

converges to 0 . And this concludes the proof of Theorem 4.1. 


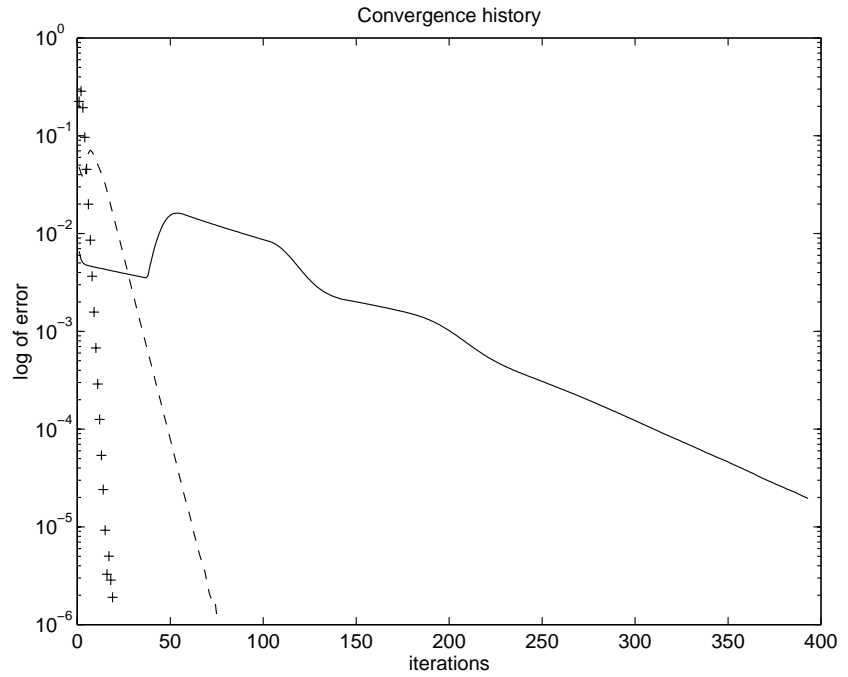

Figure 2. Convergence history curves for $\Delta t=10^{-2}(-), \Delta t=10^{-1}(--)$ and $\Delta t=1(++)$.

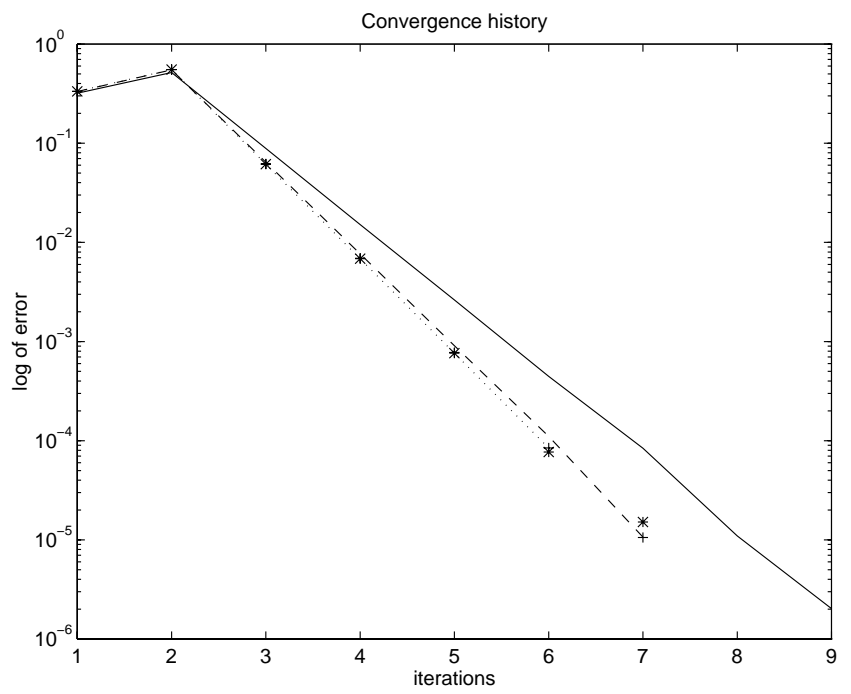

Figure 3. Convergence history curves for $\Delta t=10(-), \Delta t=10^{2}(--), \Delta t=10^{3}(++)$, $\Delta t=10^{4}(* *)$ and $\Delta t=10^{5}(\cdots)$.

\section{NUMERICAL STUDY OF THE COUPLING}

In this section we shall study numerically the convergence properties of the algorithm (41-47). This algorithm corresponds to the following systems

$$
\left\{\begin{array} { l } 
{ \frac { u _ { 1 } ^ { n + 1 } - u _ { 1 } ^ { n } } { \Delta t } + \frac { \mathrm { d } u _ { 1 } ^ { n + 1 } } { \mathrm { d } x } = a ( v _ { 1 } ^ { n + 1 } - u _ { 1 } ^ { n + 1 } ) , } \\
{ \frac { v _ { 1 } ^ { n + 1 } - v _ { 1 } ^ { n } } { \Delta t } - \frac { \mathrm { d } v _ { 1 } ^ { n + 1 } } { \mathrm { d } x } = a ( u _ { 1 } ^ { n + 1 } - v _ { 1 } ^ { n + 1 } ) , } \\
{ u _ { 1 } ^ { n + 1 } ( 0 ) = g , \quad v _ { 1 } ^ { n + 1 } ( h _ { 1 } ) = v _ { 2 } ^ { n + 1 } ( h _ { 1 } ) , }
\end{array} \quad \left\{\begin{array}{l}
\frac{u_{2}^{n+1}-u_{2}^{n}}{\Delta t}+\frac{\mathrm{d} u_{2}^{n+1}}{\mathrm{~d} x}=a\left(v_{2}^{n+1}-u_{2}^{n+1}\right), \\
\frac{v_{2}^{n+1}-v_{2}^{n}}{\Delta t}-\frac{\mathrm{d} v_{2}^{n+1}}{\mathrm{~d} x}=a\left(u_{2}^{n+1}-v_{2}^{n+1}\right), \\
u_{2}^{n+1}\left(h_{1}\right)=u_{1}^{n}\left(h_{1}\right), \quad v_{2}^{n+1}(1)=h,
\end{array}\right.\right.
$$




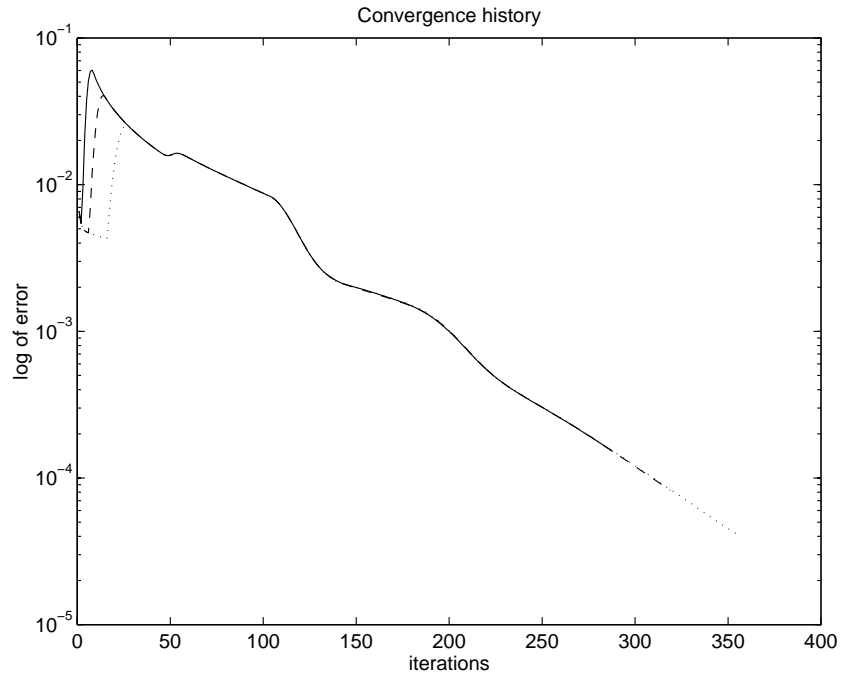

FiguRE 4. Convergence history curves for $h_{1}=0.0625(-), h_{1}=0.125(--)$ and $h_{1}=0.25(\cdots)$.

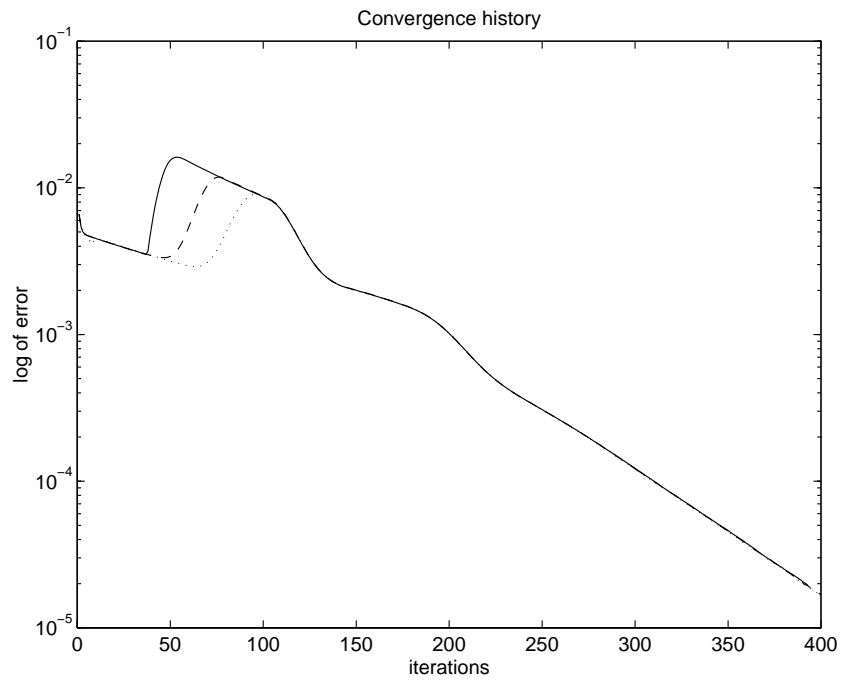

FIGURE 5. Convergence history curves for $h_{1}=0.5(-), h_{1}=0.75(--)$ and $h_{1}=0.95(\cdots)$.

and the initial conditions

$$
u_{1}^{0}=u_{10}, \quad v_{1}^{0}=v_{10}, \quad u_{2}^{0}=u_{20}, \quad v_{2}^{0}=v_{20} .
$$

For the discretization in space, we consider an equally spaced subdivision of $\left[0, h_{1}\right]$ into subintervals $\left[x_{i}, x_{i+1}\right]$, $x_{0}=0, x_{i}=x_{i-1}+\Delta x_{i}, \quad i=1, \cdots, n_{1}$, where $n_{1}$ is the number of subdivisions and $\Delta x_{i}=h_{1} / n_{1}$. We also subdivide $\left[h_{1}, 1\right]$ into equally spaced subdivision $\left[y_{i}, y_{i+1}\right], y_{0}=h_{1}, y_{i}=y_{i-1}+\Delta y_{i}, i=1, \cdots, n_{2}$, where $n_{2}$ is the number of subdivisions and $\Delta y_{i}=\left(1-h_{1}\right) / n_{2}$. 


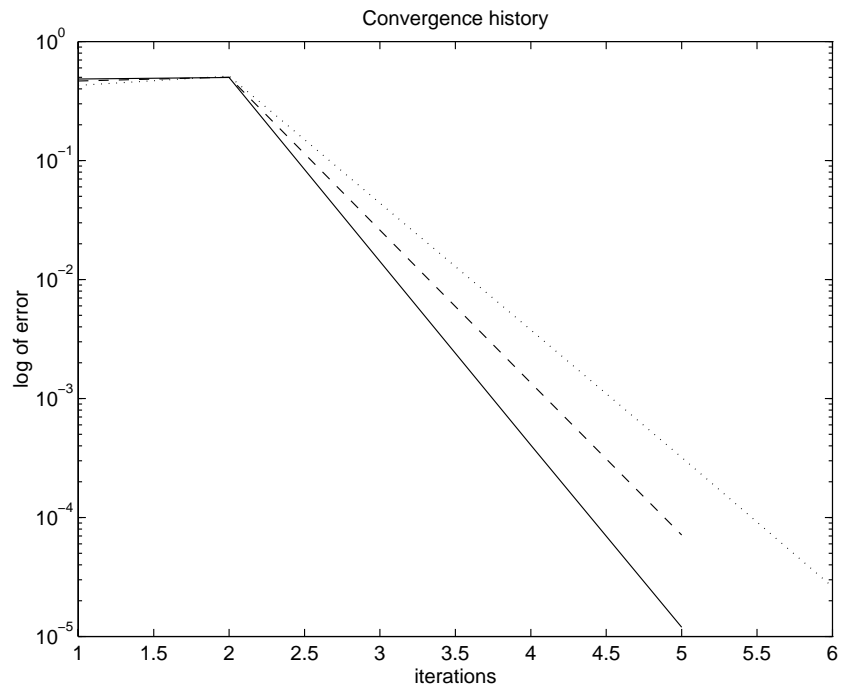

FIGURE 6. Convergence history curves for $h_{1}=0.0625(-), h_{1}=0.125(--)$ and $h_{1}=0.25(\cdots)$.

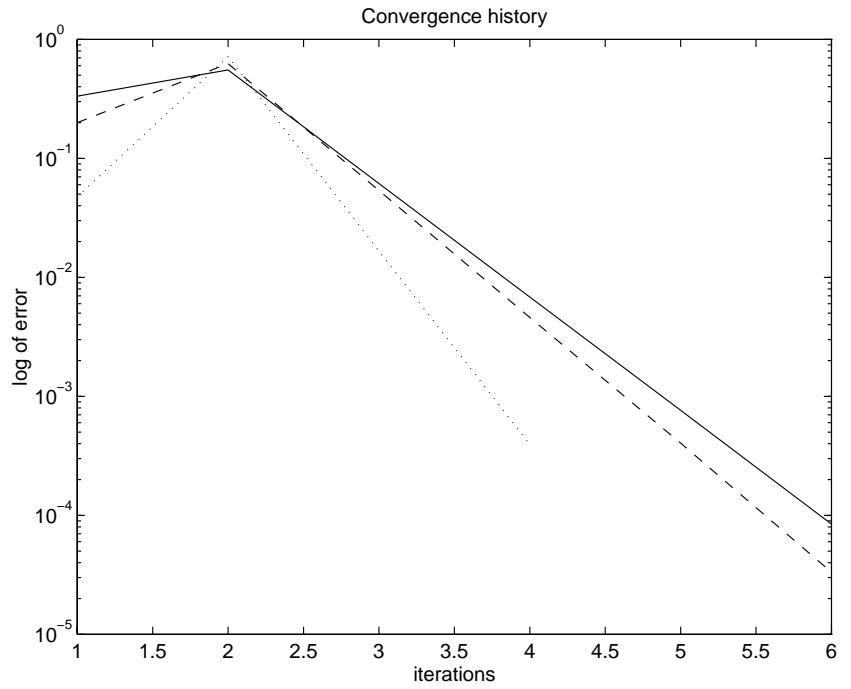

Figure 7. Convergence history curves for $h_{1}=0.5(-), h_{1}=0.75(--)$ and $h_{1}=0.95(\cdots)$.

We then use an upwinded difference method to approximate the space derivatives appearing in the algorithm. For example, for the first derivative appearing in the first equation in the algorithm, we use the approximation

$$
\frac{\mathrm{d} u_{1}^{n+1}}{\mathrm{~d} x}\left(x_{i}\right) \approx \frac{u_{1}^{n+1}\left(x_{i}\right)-u_{1}^{n+1}\left(x_{i-1}\right)}{\Delta x_{i}} .
$$

The other derivatives in the coupled problem are approximated similarly. The two problems are coupled only through their boundary conditions. The full discretization of each of these two problems leads to an algebraic system that we solve by the incomplete factorization method. 
To test the convergence properties of the algorithm, we take $a=1, h_{1}=1 / 2, g=1 / 2$, and $h=1$. We initialize the coupling algorithm by 0 on the whole interval $[0,1]$. In Figure. 1 , we show the convergence history of the algorithm for $\Delta t=0.025$. In this figure, we present the $L_{\infty}$ norm of the errors $\left\|u_{1}^{n}-u\right\|_{L^{\infty}\left[0, h_{1}\right]}$ and $\left\|u_{2}^{n}-u\right\|_{L^{\infty}\left[h_{1}, 1\right]}$ versus the iterations. The exact solution of the steady problem is given by

$$
u(x)=u(0)-\frac{u(0)-u(1)}{1+a} a x, \quad v(x)=u(x)-\frac{u(0)-u(1)}{1+a} .
$$

The plotting (not shown here) of the converged solution $u$ computed by the coupling algorithm and the exact solution $u$ of the steady Carleman equations shows no difference between these two solutions.

These numerical results clearly show that the algorithm converges and it converges to the solution of the steady Carleman equations.

In Figures 2 and 3 we show the convergence history $\left(\left\|u_{1}^{n}-u\right\|_{L^{\infty}\left[0, h_{1}\right]}\right)$ of the algorithm for a fixed $h_{1}=0.5$ and various values of $\Delta t$. In Figures 4 and 5 we show the convergence history $\left(\left\|u_{1}^{n}-u\right\|_{L^{\infty}\left[0, h_{1}\right]}\right)$ of the algorithm for a fixed $\Delta t=10^{-2}$ and various values of $h_{1}$. In Figures 6 and 7 , we show the convergence history $\left(\left\|u_{1}^{n}-u\right\|_{L^{\infty}\left[0, h_{1}\right]}\right)$ of the algorithm for a fixed $\Delta t=10^{5}$ and various values of $h_{1}$. These results show that the algorithm converges in accordance with the theory established in Section 4.

\section{REFERENCES}

[1] T. Carleman, Problèmes mathématiques dans la théory cinétique des gaz. Publ. Sc. Inst. Mittag-Leffler, Upsala (1957).

[2] E. Hille and R.S. Phillips, Functional Analysis and Semi-groups. Amer. Math. Soc. (1974).

[3] J.-F. Bourgat, P. Le Tallec and M.D. Tidriri, Coupling Navier-Stokes and Boltzmann. J. Comput. Phys. 127 (1996) $227-245$.

[4] J.-F. Bourgat, P. Le Tallec, F. Mallinger, Y. Qiu and M.D. Tidriri, Numerical coupling of Boltzmann and Navier-Stokes equations, in Proceedings Sixth I.U.T.A.M. (International Union of Theoretical and Applied Mechanics), Conference on Rarefied Flows for Reentry Problems, Marseille, France (1992).

[5] J.-F. Bourgat, P. Le Tallec, B. Perthame and Y. Qiu, Coupling Boltzmann and Euler equations without overlapping, in Domain decomposition methods in science and engineering, A. Quarteroni et al. Eds., CM 157, AMS (1994) 377-388.

[6] P. Le Tallec and M.D. Tidriri, Convergence analysis of domain decomposition algorithms with full overlapping for the advectiondiffusion problems. ICASE Report No. 96-37; Math. Comp. 68 (1999) 585-606.

[7] P. Le Tallec and M.D. Tidriri, Application of maximum principles to the analysis of a coupling time marching algorithm. J. Math. Anal. Appl. 229 158-169 (1999).

[8] P. Le Tallec and M.D. Tidriri, Kinetic upgrade of Navier-Stokes equations for transitional flows. Rapport Intermédiaire, Contrat 6465/91 ATP DPH (1992).

[9] P. Le Tallec and M.D. Tidriri, Kinetic upgrade of Navier-Stokes equations for Transitional Flows. Rapport de contrat HERMES R/Q 6465/91, INRIA (1993).

[10] A. Lukshin, H. Neunzert and J. Struckmeier, Coupling of Navier-Stokes and Boltzmann regions. Interim Report for the Hermes Project DPH 6174/91 (1992).

[11] F. Mallinger, Couplage adaptatif des équations de Boltzmann et de Navier-Stokes. Ph.D. thesis, University of Paris IX, France (1996).

[12] M.D. Tidriri, Couplage d'approximations et de modèles de types différents dans le calcul d'écoulements externes. Ph.D. thesis, University of Paris IX, France (1992).

[13] M.D. Tidriri, Domain Decomposition for Incompatible nonlinear models. INRIA Research Report RR-2378 (1994).

[14] M.D. Tidriri, Domain Decomposition for Compressible Navier-Stokes Equations with Different Discretizations and Formulations. J. Comput. Phys. 119 (1995) 271-282.

[15] M.D. Tidriri, Asymptotic analysis of a coupled system of kinetic equations. C.R. Acad. Sci. Paris Sér. I Math. 328 (1999) $637-642$. 